\title{
THE STRUCTURE OF THE COLLODION MEMBRANE AND ITS ELECTRICAL BEHAVIOR
}

IV. The Relative Merits of the Homogeneous Phase Theory and the Miceliar-Structural Theory As Applied to the

Dried Collodion Membrane

By KARL SOLLNER AND CHARLES W. CARR

(From the Department of Physiology, University of Minnesota, Minneapolis)

(Received for publication, May 25, 1942)

I

The structure of the dried collodion membrane has in the past been the subject of an extensive controversy. A great variety of arguments ${ }^{1-5}$ has been put forward to substantiate the one or the other of the two major conflicting theories, which are referred to frequently as the "solubility theory" and the "pore theory" respectively. Instead of these terms, which are rather suggestive of special mechanisms, we intend to use in this paper the more general, less suggestive terms, "homogeneous phase theory" and "structural" or preferably "micellar-structural theory" respectively.

It is not the plan of this communication to collect and re-evaluate the arguments used in this controversy. In our opinion an inadequate terminology has contributed considerably to the blurred state of the problem. However, it is not the purpose of this paper to discuss this point.

From the experimental point of view we should like to make the following remark concerning the physical aspects of the problem. It seems advisable to consider more closely the possibility of the existence of different mechanisms of permeation according to the nature of the substances which permeate a

\footnotetext{
${ }^{1}$ See, e.g., Beutner, R., Die Entstehung elektrischer Ströme in lebenden Geweben, Stuttgart, Ferdinand Enke, 1920; Beutner, R., Physical chemistry of living tissues and life processes, as studied by artificial imitation of their single phases, Baltimore, The Williams \& Wilkins Co., 1933; Beutner, R., Caplan, M., and Loehr, W. M., J. Biol. Chem., 1933, 101, 391; Beutner, R., Arch. Zellforsch., 1934, 15, 217.

${ }^{2}$ Collander, R., Kolloidchem. Beihefte, 1924, 19, 72; 1925, 20, 273; Societas Scientiarum Fennica, Commentations Biologicae II, 6, Helsingfors, 1926.

${ }^{3}$ E.g., Michaelis, L., and Perlzweig, W. A., J. Gen. Physiol., 1926-27, 10, 575; Michaelis, L., McEllsworth, R., and Weech, A. A., J. Gen. Physiol., 1926-27, 10, 671; Michaelis, L., Weech, A. A., and Yamatori, A., J. Gen. Physiol., 1926-27, 10, 685; Michaelis, L., Bull. Nat. Research Council, No. 69, 1929; Kolloid-Z., 1933, 62, 2, and other publications.

${ }^{4}$ E.g., Northrop, J. H., J. Gen. Physiol., 1928, 11, 233; 1929, 12, 435.

${ }^{5}$ E.g, Wilbrandt, W., J. Gen. Physiol., 1935, 18, 933.
} 
membrane. Closer consideration should also be given to the state of the latter; it may be that collodion membranes in the dry state behave differently (for their permeability for gases) than those in the wet state. Also the affinity of the permeating substance for the membrane material can conceivably play a much more important and characteristic rôle than generally assumed. Strong inorganic electrolytes, for example, may easily follow pathways essentially different from those which are available to compounds which in higher concentration act as swelling agents, solvents, etc. Conclusions reached on the basis of experiments with one kind of substances should not be extended without further proof to other groups. It is even conceivable that one theory may fit one set of circumstances, whereas the other theory may fit a different situation.

Before we begin to discuss the special approach to the general problem which is made in this paper, we should like to mention two independent sets of observations which, though pertinent, have been largely neglected in the literature on membranes.

It is well known that dried collodion membranes cast from the same solution may show widely varying concentration potentials even if they are cast under conditions which are kept constant as far as experimentally possible. This effect is particularly conspicuous with membranes made from electrochemically inactive preparations; here the variations in concentration potential across different membrane specimens are frequently more than 100 per cent, ${ }^{5}$ the potentials across the individual membranes being very constant and easily reproducible over long periods. (See Tables $1 a$ and $1 b$.) The homogeneous phase theory could not conceivably account for this variability of the concentration potential; in fact, it actually seems to be incompatible with it. All specimens of a homogeneous phase should have the same properties whatever their past, the more so if prepared under identical conditions. A structural theory, however, could easily accommodate the observed facts. Differences in aggregation and orientation of molecules and of micelles could easily be accounted for by uncontrollable variations in the preparation of the individual membranes. Since any structural theory necessarily assumes that orientation and aggregation are the factors which determine the structure of membranes and thus their behavior, such a theory seems greatly favored by the above mentioned variations in concentration potential.

Furthermore we feel that inadequate use has been made of the evidence in favor of the micellar-structural theory which is contained in the literature on $\mathrm{x}$-ray studies of nitrocellulose. This literature demonstrates definitely the occurrence of crystalline micelles in nitrocellulose films, the sharpness of the interferences varying greatly with different preparations. It would be attractive and profitable to discuss the extent to which $\mathrm{x}$-ray evidence indicates either a truly micellar structure or merely the presence of micelles in an amor- 
phous matrix. However, we shall limit our remarks to the statement that the micellar-structural theory receives strong support from the $\mathrm{x}$-ray literature.

II

The present work is an attempt to determine which of the two theories-the homogeneous phase theory or the micellar-structural theory-allows us to interpret the observed behavior of dried collodion membranes with solutions of strong electrolytes.

The following line of reasoning serves as a basis for our present experiments. A number of dried collodion membranes are prepared under standardized conditions from some electrochemically inactive brand of collodion (state I). The characteristic concentration potential across a representative group of these membranes is determined. Next, the membranes are "activated" by oxidation (state II) as described previously ${ }^{6}$ and the concentration potential of a representative number of these membranes is determined. The remaining majority of the membranes in state II are dissolved in the same solvent mixture as used originally. Dried collodion membranes are prepared from this solution (state III), the concentration potentials across these membranes are measured, and the results obtained in states I, II, and III are compared.

The homogeneous phase theory would predict that the properties of the membranes in state II and state III are substantially identical since the properties of an interphase which behaves like a homogeneous phase should be independent of its history. No rearrangement of the molecules, brought about by any means, should be able to alter the properties of membranes prepared from the same material.

The micellar-structural theory would predict a distinct difference between state II and state III. According to this view, the charges which are caused by chemical reactions in the membranes, or by the addition of third substances, are substantially confined to certain more accessible structural elements; i.e., the surfaces of the particles which constitute the membrane. If the membrane is dissolved, the elements which are the cause of the characteristic properties of state II are mixed with the whole mass of the membrane material. If a new membrane is now prepared from this solution, the concentration of the active groups at the accessible points is necessarily reduced as compared with state II.

The foregoing discussion is based on the tacit assumption that the interstices which govern the behavior of the membrane are larger than the minimal possible molecular interstices. Such interstices may arise due to an irregular arrangement of molecules without the formation of distinct micelles or in the formation of micelles and their arrangement. In either case the effective interstices would be of micellar as opposed to molecular magnitude, and either

${ }^{6}$ Sollner, K., Abrams, I., and Carr, C. W., J. Gen. Physiol., 1941, $25,7$. 
case would fulfill the necessary condition for the above considerations. We designate them as having a "micellar-structural" constitution, as opposed to the molecular-structural constitution of a crystal or liquid, the emphasis, as expressed in our terminology, is more on the nature of the interstices than on the structure-forming physical elements. That the formation of micelles, at least in a rudimentary manner, is $a$ priori the more likely situation with the fibrous nitrocellulose molecules is here without particular importance. The homogeneous phase theory, of course, must necessarily assume a molecularstructural constitution of a crystalline or glasslike liquid character with true equivalence, or at least statistical equivalence, for all actual or virtual interstices. The behavior to be expected from a membrane having such a molecular-structural constitution was pointed out in a preceding paragraph.

\section{III}

A necessary prerequisite of the experiment, of course, is that the activation is not too thorough. If the number of active groups which are introduced in state II is so great that too many of them come to lie in the critical spots in state III, the results may become less pronounced. In order to demonstrate the anticipated effect most clearly, the activation should be stopped just at the time when maximal or nearly maximal concentration potentials are obtained. A more prolonged oxidation would necessarily lead to a gradual diminution in the expected effect. One further has to ascertain that a significant loss of active material does not occur at any state of the whole experiment; otherwise its purposeful evaluation is impossible.

Below we report two series of experiments carried out according to the plan outlined. In the one series (series $a$ ), the degree of oxidation applied is about the minimal which yields membranes of high activity; series $b$ was subjected to a much stronger oxidation.

For the preparation of the original membranes (state I), Mallinckrodt "Parlodion" purified by boiling 7 hours with 90 per cent alcohol" was used; this purified material has been shown to be the least active of the available collodion preparations. $5 \mathrm{gm}$. per $100 \mathrm{ml}$. were dissolved in a mixture of 25 per cent absolute alcohol and 75 per cent absolute ether, this mixture being adhered to throughout all the work reported in this paper.

For each of the two experimental series, 70 bag-shaped membranes were cast by hand in $22 \times 85 \mathrm{~mm}$. pyrex test tubes and allowed to dry in the air at room temperature for 48 hours. After 48 hours the membranes are perfectly dry and can be removed with water from the test tubes; the uneven, frequently heavy rims of the membranes are cut off. The average thickness of these membranes is about $10 \mu$, as calculated from area and weight, the specific gravity of collodion being 1.6. Appreciably thinner and thicker spots are found on

\footnotetext{
${ }^{7}$ Sollner, K., Carr, C. W., and Abrams, I., J. Gen. Physiol., 1942, $25,411$.
} 
each individual membrane. Out of each group of 70 membranes (state I), every seventh was picked to obtain a representative group of ten membranes for the determination of the characteristic concentration potentials. For these measurements the membranes were fitted loosely over glass rings, held in wooden test tube clamps, and filled about two-thirds with 0.1 molar potassium chloride solution. They were immersed to the same level in 0.01 molar potassium chloride solution (temperature about $25^{\circ} \mathrm{C}$.). Potential measurements were made about 5 minutes after the membranes were interposed between the potassium chloride solutions and repeated afterwards to ascertain

TABLE I

The Concentration Potential 0.1 \& $\mathrm{KCl} / 0.01$ \& $\mathrm{KCl}$ across Dried Collodion Membranes Prepared from Purified "Parlodion" (State I), Potentials in Millivolts

\begin{tabular}{|c|c|c|c|c|c|}
\hline \multicolumn{3}{|c|}{$\stackrel{a}{\stackrel{a}{(S e r i e s} a)}$} & \multicolumn{3}{|c|}{ (Series $b$ ) } \\
\hline \multirow{2}{*}{$\begin{array}{l}\text { Membrane } \\
\text {. }\end{array}$} & \multicolumn{2}{|c|}{ Concentration potential } & \multirow{2}{*}{ Membrane } & \multicolumn{2}{|c|}{ Concentration potential } \\
\hline & $5 \mathrm{~min}$ & 2 days & & $5 \mathrm{~min}$. & 2 days \\
\hline & mv. & mv. & & $m v$. & $m v$. \\
\hline 1 & 35 & 36 & 71 & 26 & $(8)^{*}$ \\
\hline 8 & 28 & 26 & 78 & 34 & 35 \\
\hline 15 & 28 & 27 & 85 & 31 & 33 \\
\hline 22 & 27 & 25 & 92 & 30 & 35 \\
\hline 29 & 37 & 38 & 99 & 29 & 29 \\
\hline 36 & 32 & 33 & 106 & 26 & 28 \\
\hline 43 & 20 & 21 & 113 & 37 & 37 \\
\hline 50 & 13 & 15 & 120 & 36 & 36 \\
\hline 57 & 33 & 33 & 127 & 29 & 28 \\
\hline 64 & 22 & 22 & 134 & 26 & 27 \\
\hline Mean. & 27.5 & 27.6 & Mean. & 30.4 & 32.0 \\
\hline
\end{tabular}

* This membrane was damaged when the potential measurement was made. The value in parenthesis is not considered in the calculation of the mean.

the stability of the potential. The 5 minute value was found to be the final stable potential in nearly all cases. The accuracy of these determinations is $\pm 1 \mathrm{mv}$. On account of the high ohmic resistance of the membranes in state $\mathrm{I}$, it was convenient to use the volt scale of a commercial (Leeds and Northrup) glass electrode set, in spite of its limited ( $\pm 1 \mathrm{mv}$.) accuracy.

The two series of ten membranes each in state I were kept in distilled water (thymol being present as a preservative) and the concentration potential measured again after 2 days. The potential measurements performed with the membranes in state $I$ are summarized in Tables $I a$ and $I b$; the mean values for the two series are 27.5 and $30.4 \mathrm{mv}$. respectively, the difference being statistically insignificant. 
The remaining two sets of 60 membranes each were oxidized under the following conditions; series $a, 3$ hours oxidation time in a 0.05 molar $^{8}$ sodium hypobromite solution at $\mathrm{pH} 6$; series $b, 1$ hour in molar ${ }^{8}$ hypobromite solution at the same $\mathrm{pH}$.

TABLE II

The Concentration Potential $0.1 \mathrm{M} \mathrm{KCl} / 0.01 \mathrm{M} \mathrm{KCl}$ across Dried Collodion Membranes Activated by Oxidation with Sodium Hypobromite Solution (State II), Potentials in Millivolts

\begin{tabular}{|c|c|c|c|c|c|}
\hline \multicolumn{3}{|c|}{$\begin{array}{c}a \\
\text { (Series } a ; 3 \text { hrs. oxidation with } 0.05 \mathrm{M} \\
\text { potassium hypobromite solution) }\end{array}$} & \multicolumn{3}{|c|}{$\begin{array}{l}\text { (Series } b ; 1 \text { hr. oxidation with } 1.0 \mathrm{M} \\
\text { potassium hypobromite solution) }\end{array}$} \\
\hline \multirow{2}{*}{ Membrane } & \multicolumn{2}{|c|}{ Concentration potential } & \multirow{2}{*}{ Membrane } & \multicolumn{2}{|c|}{ Concentration potential } \\
\hline & $5 \mathrm{~min}$. & 2 days & & $5 \mathrm{~min}$. & 2 days \\
\hline & $m v$. & $m v$ & & $m v$. & $m v$. \\
\hline 2 & 50 & $(0)^{*}$ & 72 & 52 & 52 \\
\hline 3 & 50 & 50 & 73 & 52 & 49 \\
\hline 9 & 50 & $(0)^{*}$ & 79 & 50 & 48 \\
\hline 10 & 50 & 50 & 80 & 52 & 51 \\
\hline 16 & 50 & 48 & 86 & 51 & 50 \\
\hline 17 & 50 & 50 & 87 & 50 & 49 \\
\hline 23 & 50 & 50 & 93 & 53 & 51 \\
\hline 24 & 50 & 49 & 94 & 53 & 51 \\
\hline 30 & 50 & 47 & 100 & 50 & 50 \\
\hline 31 & 50 & 50 & 101 & 52 & 51 \\
\hline 37 & 50 & 50 & 107 & 53 & 51 \\
\hline 38 & 49 & 45 & 108 & 52 & 50 \\
\hline 44 & 50 & $(0)^{*}$ & 114 & 51 & 50 \\
\hline 45 & 50 & 50 & 115 & 52 & 50 \\
\hline 51 & 50 & 50 & 121 & 52 & 51 \\
\hline 52 & 50 & 50 & 122 & 50 & 49 \\
\hline 58 & 50 & $(0)^{*}$ & 128 & 51 & 51 \\
\hline 59 & 50 & 48 & 129 & 51 & 50 \\
\hline 65 & 49 & 50 & 135 & 50 & 50 \\
\hline 66 & 50 & 50 & 136 & 50 & 51 \\
\hline Mean. & 49.9 & 49.2 & Mean. . & 51.3 & 50.2 \\
\hline
\end{tabular}

* See footnote to Table I.

After oxidation the membranes were washed thoroughly with distilled water with frequent changes of water. Out of each of the two groups of $60 \mathrm{mem}$ branes, 20 membranes were picked as indicated in Table II. These membranes were considered as representative of the two groups; the characteristic con-

${ }^{8}$ The term "molar" refers here to the concentration of the sodium hydroxide solution from which the hypobromite solution is prepared by the addition of bromine (Sollner, K., Abrams, I., and Carr, C. W., J. Gen. Physiol., 1941, 25, 7). 
centration potentials obtained with them after about 5 minutes (as before) are listed in Table II $a$ and $\Pi \mathrm{I} b$ The tables also give the mean potential values, $49.9 \mathrm{mv}$. for the less thoroughly oxidized series and $51.3 \mathrm{mv}$. for the more strongly oxidized membranes.

TABLE III

The Concentration Potential 0.1 M KCl/0.01 $\mathrm{M} \mathrm{KCl}$ across Dried Collodion Membranes Prepared from Redissolved Activated Membranes (State III), Potentials in Millivolts

\begin{tabular}{|c|c|c|c|c|c|}
\hline \multicolumn{3}{|c|}{ (Series $a$ ) } & \multicolumn{3}{|c|}{$\stackrel{b}{b}$} \\
\hline \multirow{2}{*}{ Membrane } & \multicolumn{2}{|c|}{ Concentration potential } & \multirow{2}{*}{ Membrane } & \multicolumn{2}{|c|}{ Concentration potential } \\
\hline & $5 \mathrm{~min}$. & 2 days & & $5 \mathrm{~min}$. & 2 days \\
\hline & $m v$. & mข. & & mv. & mo. \\
\hline a & 32 & 33 & A & 41 & 40 \\
\hline b & 33 & 33 & B & 38 & 36 \\
\hline c & 20 & 22 & C & 49 & 48 \\
\hline d & 26 & 25 & D & 34 & 34 \\
\hline e & 22 & 22 & $\mathbf{E}$ & 50 & 48 \\
\hline $\mathbf{f}$ & 28 & 28 & $\mathbf{F}$ & 49 & $(31)^{*}$ \\
\hline g & 35 & 41 & $\mathbf{G}$ & 18 & 20 \\
\hline $\mathbf{h}$ & 13 & 13 & $\mathbf{H}$ & 45 & 45 \\
\hline $\mathbf{i}$ & 28 & 29 & I & 22 & 22 \\
\hline j & 28 & 28 & J & 40 & 40 \\
\hline $\mathbf{k}$ & 16 & 16 & $\mathbf{K}$ & 32 & 36 \\
\hline 1 & 28 & 29 & $\mathrm{~L}$ & 40 & 41 \\
\hline \multirow[t]{8}{*}{ m } & 28 & 30 & $\mathbf{M}$ & 39 & 39 \\
\hline & & & $\mathbf{N}$ & 12 & 13 \\
\hline & & & $\mathbf{O}$ & 44 & 44 \\
\hline & & & $\mathbf{P}$ & 47 & 48 \\
\hline & & & $\mathbf{Q}$ & 40 & $(31)^{*}$ \\
\hline & & & $\mathbf{R}$ & 20 & 20 \\
\hline & & & $S$ & 47 & 47 \\
\hline & & & $\mathbf{T}$ & 47 & 48 \\
\hline Mean... & 26.0 & 26.8 & Mean. & 37.7 & 37.2 \\
\hline
\end{tabular}

* See footnote to Table I.

After the potential measurements were made, these membranes were washed in distilled water and kept for 2 days in distilled water (with thymol). The concentration potentials were measured again after this time. No significant amount of material which causes the electro-chemical behavior of the membranes has been washed out during this 2 day period.

The 40 remaining membranes in state II of each of the two series were airdried and dissolved in the standard ether-alcohol mixture. Membranes were cast from the resulting solution and dried (state III) as described before, each 
solution allowing the preparation of only a limited number of membranes. These membranes in state III were removed from the glass tubes and the concentration potentials were measured after about 5 minutes contact with the electrolyte solutions. The measurements were repeated after 2 days, as before, to determine whether any change in activity occurs. This, however, was not the case. The thickness of these membranes averaged about $10 \mu$ as before. Table III $a$ and III $b$ give the potential measurements of the membranes in state III pertaining to the two series of experiments. A few obviously damaged membranes giving zero or very low potentials are not recorded in the table. The mean values in state III are 26.0 and $37.7 \mathrm{mv}$. respectively.

It is hardly necessary to point out that the moderate oxidation in series $a$ gives the more clear cut results, as compared with series $b$ in which the oxidation was more thorough. We may add that a third series of analogous experiments was carried out in which the oxidation was still more thorough than in series $b$. In agreement with expectation, the average concentration potential in state III was in this case rather high, about $49 \mathrm{mv}$.

It may be worth-while to record the fact that the membranes in state III behave like normal dried collodion membranes prepared from inactive collodion. The membranes of state III of series $a$ after thorough oxidation gave in this new state (state IV) high characteristic concentration potentials, the mean value being slightly higher than $51 \mathrm{mv}$.

\section{IV}

The experimental results recorded in Tables I, II, and III are so unequivocal, as to necessitate hardly any discussion. The concentration potentials obtained in state III are much lower than in state II, the concentration potential in state III in our series $a$ being actually identical (within experimental accuracy) with that of state I. If we recall the previously outlined predictions of the "homogeneous phase" and the "micellar-structural" theories, we see immediately that the experimental results agree excellently with the predictions of the micellar-structural theory; they are incompatible with the predictions and therefore with the basic assumptions of the homogeneous phase theory.

Micellar and not molecular interstices are the factors governing the behavior of dried collodion membranes-at least in the case of strong inorganic electrolytes. This, of course, does not imply that molecular interstices do not exist; they must necessarily exist. It means only that their existence is not an important factor in the characteristic membrane behavior; this is true even if it should turn out that the total volume of the molecular interstices exceeds the total volume of the micellar interstices. There is no indication that all the micellar interstices are of the same size or that they are equally accessible; 
rather, non-equivalence in both respects has to be expected as indicated previously ${ }^{7}$ for the case of the porous collodion membrane. Some direct experimental evidence as to the non-equivalence of the micellar interstices is already contained in the literature. ${ }^{9}$ It seems probable that a mere fraction of the micellar interstices, the readily accessible ones, determine the characteristic membrane behavior. The obvious methods of attacking these and similar problems involve quantitative studies of the rates of water and electrolyte uptake by the membranes and of the final equilibrium. These studies must be combined with conductance and concentration potential measurements. However, all such studies depend upon information upon the water uptake and swelling of the dried collodion membrane. Such studies are under way at this time.

\section{SUMMARY}

1. Experiments were carried out to decide whether a homogeneous phase (solubility) theory or a micellar-structural theory more adequately describes the behavior of dried collodion membranes with solutions of strong electrolytes.

2. A number of dried collodion membranes were prepared from an electrochemically inactive collodion preparation (state I); the characteristic concentration potentials across them were low, about $30 \mathrm{mv}$. The membranes were activated by oxidation (state II) to give maximum or nearly maximum concentration potentials (about $50 \mathrm{mv}$.). The oxidized membranes are dried, dissolved in alcohol-ether, and a new set of dry collodion membranes prepared from this solution (state III). The concentration potentials across these membranes are low.

3. Since the properties of a homogeneous phase should not be influenced by a rearrangement of its constituent particles, the experimental results do not support a homogeneous phase (solubility) theory, but they agree with the predictions of the micellar-structural theory. The characteristic behavior of dried collodion membranes in solutions of strong inorganic electrolytes is therefore due to the micellar character of its interstices.

${ }^{9}$ Green, A. A., Weech, A. A., and Michaelis, L., J. Gen. Physiol., 1929, 12, 473. 